\title{
Non-small cell lung cancer: the era of targeted therapy
}

This article was published in the following Dove Press journal:

Lung Cancer:Targets and Therapy

2 July 2012

Number of times this article has been viewed

\section{Mara B Antonoff Jonathan D'Cunha}

Division of Thoracic and Foregut Surgery, Department of Surgery, University of Minnesota,

Minneapolis, MN, USA
Correspondence: Jonathan D'Cunha Division of Thoracic and Foregut Surgery, Department of Surgery, University of Minnesota, 420 Delaware Street SE, Mayo Mail Code 207, Minneapolis, MN 55455, USA

Tel + I 6126243277

Fax + I 6I26259657

Email jdcunha@umn.edu
Abstract: In this review, the authors aim to provide an overview of current molecular targeted therapies for NSCLC, to propose an algorithm for clinical application of presently available treatment strategies, and to identify future directions for this important area of research. Historically, choice of treatment algorithm for the management of non-small cell lung cancer (NSCLC) has relied heavily upon histology and clinical staging information, typically assigning patients to surgery, chemotherapy, radiation, or a combination thereof. However, previous treatment strategies have been fraught with disappointing response rates and significant systemic toxicities. The concept of personalized therapy for NSCLC involves characterization of each individual patient's tumor, in terms of genetic aberrations and expected biologic behavior, and using this information to tailor subsequent clinical management. Several driver mutations have been identified to date in subsets of patients with NSCLC, and, by focusing on specific molecular targets, new agents have been developed with the intent of treating the cancer cells while causing minimal toxicity to benign, healthy cells. In particular, current strategies exist to identify patients with epidermal growth factor receptor gene mutations and anaplastic lymphoma kinase rearrangements, with promising results upon clinical application of agents targeting these abnormalities. Moving forward, attempts are being made to determine comprehensive genetic and biologic characterization of individuals' NSCLC tumors and to incorporate these findings into everyday practice. The era of targeted therapy is upon us. As we seek to expand our knowledge of the specific molecular and cellular derangements leading to growth and proliferation of NSCLC tumors, our efforts bring us closer to ultimately providing each patient with a personalized plan of care.

Keywords: personalized therapy, algorithm, EGFR, ALK, KRAS

\section{Introduction}

Lung cancer remains the number-one worldwide cause of cancer-related mortality. In the United States alone, the annual number of deaths due to lung cancer is predicted to reach 160,000 in 2012, with more than 225,000 expected new cases. ${ }^{1}$ Accounting for more predicted deaths than malignancies of the breast, prostate, colon and rectum, and pancreas combined, lung cancer continues to take an enormous toll - in terms of both health care expenses incurred and lives lost. Non-small cell lung cancer (NSCLC) - comprised of squamous cell carcinoma, adenocarcinoma, and large-cell carcinoma - accounts for approximately $85 \%$ of all primary lung cancers. ${ }^{2}$ The majority of individuals with NSCLC are deemed inoperable upon initial evaluation, consequent to the presence of locally advanced or metastatic disease. ${ }^{3}$ Despite considerable efforts put toward improving outcomes for patients with NSCLC, reported 5-year survival remains in the range of $15 \%$ for all-comers and less than $4 \%$ for patients with distant 
disease, and, unfortunately, minimal improvement in these dismal statistics over the last 3 decades. ${ }^{1,4}$

In the United States, cigarette smoking remains the strongest risk factor for the development of lung cancer, such that the risk of dying of lung cancer for an individual who has smoked a pack per day for 20 years is nearly 19 times higher than the risk for a nonsmoker. ${ }^{5}$ Recent data suggest that approximately $20 \%$ of adults in the United States currently smoke, with former smokers accounting for an additional $20 \%$ of the populace at large and nearly $40 \%$ of new lung cancer diagnoses. ${ }^{6}$ For those who have successfully overcome the habit, an elevated risk of lung cancer persists even beyond a decade after quitting. ${ }^{7}$ Approximately $15 \%$ of new lung cancer diagnoses in the United States occur in never-smokers. ${ }^{7}$ This group of individuals is, in some ways, of particular interest to researchers; with unclear and oftentimes unidentifiable environmental stimuli for tumor development, never-smokers are more likely to have tumors with specific molecular characteristics and may be candidates for new lines of targeted therapy. 8,9

\section{Current management of NSCLC}

For new patients presenting with NSCLC, the typical algorithm of disease management consists of initial diagnosis following work-up for concerning symptoms or an incidentally identified lesion. Tissue diagnosis may be achieved via one of several approaches, including imageguided percutaneous biopsy, bronchoscopic evaluation, or surgical biopsy. ${ }^{4}$ After confirmation of cancer diagnosis, patients go on to staging, at which time they are assessed in terms of both lymph node status and presence of distant metastases. This process, too, may involve a combination of modalities, including imaging studies and invasive procedures. ${ }^{4}$ Relying heavily on the pathologic diagnosis and clinical stage assignment, and frequently involving a multidisciplinary team of health care providers, a treatment plan is established, ultimately consisting of surgery, chemotherapy, radiation, or a combination thereof. A given patient may not necessarily be an appropriate candidate for the algorithmically assigned treatment plan, depending on comorbidities, functional status, and willingness to accept the associated side effects of the various treatment modalities.

Surgical resection is the standard of care for early stage NSCLC, with lobectomy considered the operation for optimal oncologic outcome. ${ }^{10}$ While this dictum has been questioned of late, remaining a controversial topic and an ongoing area of interest among surgeons, the role of operative resection overall is fairly clear. ${ }^{6,11}$ However, despite the efficacy of surgical intervention for early-stage lung cancer, only $16 \%$ of new lung cancer cases are diagnosed with localized disease that is potentially curable, thus contributing significantly to the dismal outcomes from this disease. ${ }^{4}$

For those patients who present with locally advanced or metastatic NSCLC, palliative chemotherapy is associated with only modest survival prolongation and indeterminate impact on quality of life. ${ }^{12}$ Platinum-based chemotherapy has become the standard of care; however, improvement in outcome from such pharmacologic agents has been modest at best, with reported 5-year survival advantages in the range of $4 \%-15 \% .{ }^{13}$ The current first-line therapy for those patients with advanced NSCLC consists of a platinum agent - either cisplatin or carboplatin - administered in combination with one of several different chemotherapeutic drugs, including gemcitabine, paclitaxel, or vinorelbine. ${ }^{14}$ Response rates to these regimens are in the range of $17 \%-32 \%{ }^{15}$ For those who fail platinum-based therapy, because of either intolerance or lack of response, current second-line treatment consists of docetaxel. ${ }^{16}$ Pemetrexed is an alternative second-line agent, with similar efficacy to docetaxel and potentially fewer side effects. ${ }^{17}$

Patients with advanced NSCLC unresponsive to all lines of chemotherapy can, at times, be treated with radiation in order to decrease tumor size and associated symptoms. Goals of care, at this point, are often turned toward psychosocial support and alleviation of discomfort. ${ }^{18}$ Aside from palliation in advanced disease, radiation may also be used concurrently with chemotherapy as definitive treatment for disease that is not amenable to surgical intervention, as adjuvant therapy for positive resection margins, or in treating nodal fields felt to be at high risk for regional recurrence. ${ }^{4}$

In recent years, additional therapeutic options have come into the mix. Efforts have been put toward the recognition of specific driver mutations - those alterations that foster neoplastic transformation and contribute to tumor progression. Several driver mutations have been identified in subsets of patients with NSCLC, and, by focusing on specific molecular targets, new agents have been developed with the intent of treating the cancer cells with minimal toxicity to normal cells in the body. However, at this time, such options are limited and pertain to a very select group of patients.

\section{Ideal management of NSCLC}

The "holy grail" for management of patients with NSCLC would rely upon identification of an individual's specific tumor characteristics upon initial presentation. Diagnosis would involve not only confirmation of pathology but also 
elucidation of the exact gene expression and regulatory derangements that enable tumor growth and progression. Such information would provide each patient with a personalized approach, rather than a formulaic algorithm, and would afford thoracic oncologists the opportunity to intelligently and strategically target each individual's tumor.

Authors of a number of previous studies have sought to characterize the molecular changes occurring in the processes of NSCLC tumorigenesis and metastasis, with significant attention directed toward preclinical approaches utilizing both genomic and proteomic analyses for the identification of potentially useful tumor targets. ${ }^{19}$ Such approaches are of unquantifiable benefit to patients. As outlined earlier, improvements in outcome from standard chemotherapeutic regimens have been disappointing. Furthermore, the side effect profiles of such medications are significant, frequently resulting in substantial symptoms and serious morbidities. Also, as with any therapy offered to patients, we must carefully weigh both the risks and the benefits prior to executing a treatment plan. With the concept of individualized, customizable therapy for all patients, we can aim to reserve specific therapies for those patients whose tumors will have the greatest responsiveness to the agent in question, improving overall efficacy and limiting futile treatment efforts. ${ }^{20}$

Current chemotherapeutic regimens use broad strokes to take down a wide spectrum of cell types, with even the best-tolerated agents invariably resulting in unavoidable side effects upon toxicity to normal, benign cells. As the notion of personalized therapy has come into popularity, attention has been directed toward limiting the toxicity of a given chemotherapeutic agent to those individuals with the greatest likelihood of responding to such a drug. This concept is exemplified by studies investigating the utility of platinumbased chemotherapies in groups of patients stratified by specific biomarkers in attempts to predict oncologic response. The International Adjuvant Lung Cancer Trial Biology study analyzed 761 NSCLC tumors, using immunohistochemical staining to determine the expression of the excision repair cross-complementation group 1 (ERCC1) protein in operative specimens. ${ }^{21}$ Results of this study demonstrated that patients with completely resected ERCC1-negative tumors benefited from cisplatin-based adjuvant chemotherapy, whereas patients with ERCC1-positive tumors did not. Such breakthrough studies are highly useful in defining who might best be served by traditional chemotherapy; however, we may be able to do much better than traditional chemotherapy.

Thinking bigger, the ultimate goal would be to move beyond such broadly striking chemotherapeutic agents, with the ability to identify the "haywire" circuitry in every patient's malignancy, offering each individual a magic bullet, or a combination of magic bullets, for his or her diagnosed molecular abnormality.

Targeted therapy is a cognitive step in the right direction. However, one must further consider the likelihood that (a) tumor cells may have more than one molecular derangement and (b) tumor cells possess a great deal of adaptability. Focusing on one problem may simply trigger the cells to work around the roadblock and proliferate via a different point in the process. Consequently, the ideal strategy would be to design a combination of agents, a "magic bullet cocktail," so to speak, designed for each individual, so as to provide the patient with a personalized, highly efficacious, and systemically nontoxic plan of care.

\section{What do we know now?}

For over a decade, researchers and clinicians have had remarkable success in utilizing genotype-based therapy for patients with malignancies such as chronic myelogenous leukemia and gastrointestinal stromal tumors, with tumortargeting agents demonstrating greater efficacy and fewer side effects than traditional chemotherapy. ${ }^{22,23}$ Interest in applying such strategies to the treatment of NSCLC has been fervent, and the shifting paradigm has already dramatically affected lung cancer treatment. ${ }^{24}$ Unfortunately, however, consideration of our present depth of knowledge is humbling. While great strides have clearly been made with several landmark studies, we are still only able to alter the natural course of this lethal disease for the small fraction of our patients whose tumors possess one of the few mutations that are presently known and targetable.

Our knowledge is limited, but we are getting better. Recent studies estimate that approximately $50 \%-60 \%$ of patients with NSCLC have at least one identifiable driver mutation, with the most common mutations being in the Kirsten ras (KRAS) gene (24\%) and the epidermal growth factor receptor (EGFR) gene (13\%-22\%), with translocations involving anaplastic lymphoma kinase $(A L K)$ in another $5 \%-6 \%$ (Figure 1). ${ }^{24,25}$ Several additional mutations have been identified among patients with NSCLC; unfortunately, however, while these abnormalities may serve as therapeutic targets in the future, specific drugs have not yet been developed. Great efforts are put toward identifying patients with abnormalities in $E G F R$ and $A L K$, as agents aimed at these targets are currently available (Table 1). However, again, this provides no aid to the majority of patients presenting to clinicians with new diagnoses of NSCLC. 


\section{Frequency of gene mutations in NSCLC}

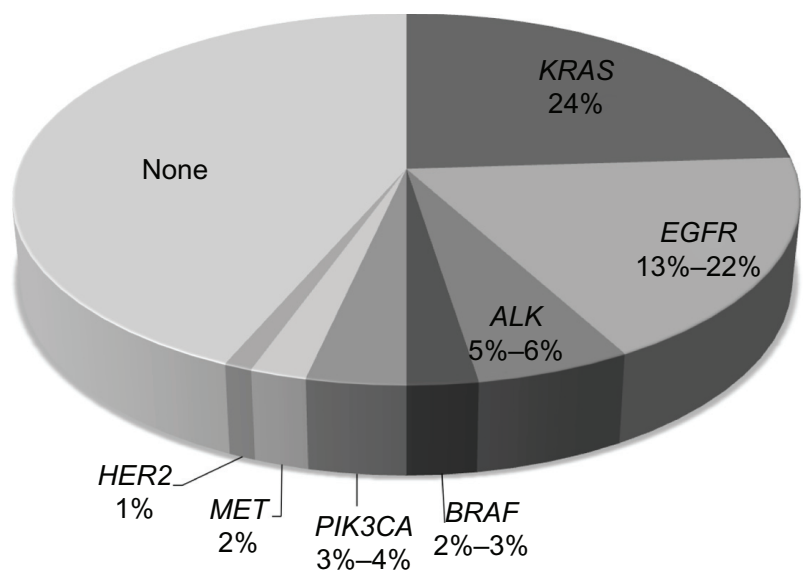

Figure I Frequency of gene mutations in non-small cell lung cancer (NSCLC). Note: The most commonly identified mutations are in the epidermal growth factor receptor (EGFR) gene and the Kirsten ras (KRAS) gene, with a large number of patients still without identifiable targets for therapy.

Abbreviations: ALK, anaplastic lymphoma kinase; HER2, human epidermal growth factor receptor 2; MET, mesenchymal-epithelial transition; PIK3CA, phosphoinositide3-kinase, catalytic, alpha polypeptide.

\section{EGFR}

EGFR, like other members of the human epidermal growth factor receptor (HER) family, is a transmembrane tyrosine kinase receptor expressed on epithelial cells throughout the body. Upon extracellular ligand binding, EGFR either forms a homodimer or heterodimerizes with another member of the HER family, resulting in tyrosine autophosphorylation and subsequent kinase activation, ultimately leading to phosphorylation of target proteins and downstream signaling. In healthy epithelial cells, these downstream functions include, among others, cellular growth and differentiation. ${ }^{26}$ EGFR mutations have been observed in NSCLC - specifically, adenocarcinoma - resulting in dysregulated cellular growth and proliferation. ${ }^{27}$ It is on the basis of these findings that EGFR tyrosine kinase inhibitors (TKIs) (erlotinib and gefitinib) have been utilized as part of treatment strategies for appropriate patients with NSCLC. Early successes with this strategy have led to some authors referring to EGFR-directed therapy as the "poster child" for targeted therapy in lung cancer. ${ }^{28}$ Patient selection, however, is key. The presence of an EGFR mutation strongly predicts likelihood of response to TKI therapy, with an observed response rate of about $80 \%$ among individuals whose tumors harbor the mutation and only $10 \%$ among those whose tumors do not. ${ }^{4,29}$

The first EGFR TKI to be evaluated in the treatment of NSCLC was gefitinib. Based on the results of two randomized phase II trials (IDEAL [Iressa Dose Evaluation in Advanced Lung Cancer] 1 and 2 trials) evaluating the efficacy of this drug as a second- and third-line agent for NSCLC, it received rapid approval from the US Food and Drug Administration in 2003 as a third-line agent for advanced NSCLC. ${ }^{30,31}$ In these studies, overall response rates of patients (not previously selected for EGFR mutations) were from $9 \%$ to $19 \%$, rendering this compound of significant interest. Erlotinib, an orally administered TKI, was subsequently developed and investigated for use in NSCLC. The BR-21 landmark study, a randomized, double-blind trial conducted by the National Cancer Institute of Canada Clinical Trials Group, compared erlotinib with placebo after failure of first-line, standard chemotherapy for NSCLC. ${ }^{32}$ By following more than 700 patients with advanced (stage IIIb/IV) disease, investigators proved the utility of erlotinib in terms of prolonged survival (6.7 versus 4.7 months for erlotinib and placebo, respectively; $P<0.001) .{ }^{32}$ Further studies have since demonstrated the efficacy of these drugs in achieving an overall clinical response

Table I Treatments to target molecular aberrations in non-small cell lung cancer (NSCLC)

\begin{tabular}{|c|c|c|c|c|}
\hline $\begin{array}{l}\text { Molecular } \\
\text { aberration }\end{array}$ & $\begin{array}{l}\text { Frequency } \\
\text { in NSCLC }\end{array}$ & Therapeutic target & Sample agent & State of progress \\
\hline \multirow[t]{2}{*}{ EGFR mutations } & $13 \%-22 \%$ & Tyrosine kinase inhibitors & Gefitinib, erlotinib & $\begin{array}{l}80 \% \text { response among patients with } \\
\text { mutation; found to decrease tumor burden } \\
\text { and prolong progression-free and overall } \\
\text { survival }\end{array}$ \\
\hline & & EGFR monoclonal antibodies & Cetuximab & $\begin{array}{l}\text { Survival advantage when added to } \\
\text { traditional chemotherapy; further } \\
\text { studies underway with matuzumab and } \\
\text { nimotuzumab }\end{array}$ \\
\hline EML4-ALK fusion & $5 \%-6 \%$ & ALK inhibitors & Crizotinib & $\begin{array}{l}\text { Tumor regression and disease control in } \\
\text { preliminary studies; phase III trial underway }\end{array}$ \\
\hline KRAS & $24 \%$ & $\begin{array}{l}\text { Small molecule inhibitors, inhibition } \\
\text { of downstream effector pathways }\end{array}$ & $\mathrm{N}-\mathrm{A}$ & $\begin{array}{l}\text { Potential therapies under preclinical } \\
\text { investigation; no current clinical trials }\end{array}$ \\
\hline
\end{tabular}

Abbreviations: ALK, anaplastic lymphoma kinase; EGFR, epidermal growth factor receptor; EML4-ALK, echinoderm microtubule-associated protein-like 4 and anaplastic lymphoma kinase; KRAS, Kirsten ras; N/A, not applicable. 
superior to that of traditional chemotherapy and improved quality of life when given to patients with EGFR mutations and advanced disease. ${ }^{3-35}$ A phase III trial comparing gefitinib and carboplatin-paclitaxel therapy in 230 patients with advanced NSCLC who were selected for EGFR mutations was stopped early after a planned interim analysis demonstrated a significant difference in progression-free survival between the two treatment arms $(P<0.001) .{ }^{34}$ In this study, after an average follow-up period of 17 months, investigators found that $73.7 \%$ of patients receiving the EGFR TKI had responded clinically, while only $30.7 \%$ of the patients receiving standard chemotherapy did so. As a result of its efficacy in these situations, its ease of administration, and its limited systemic toxicity, erlotinib is considered first-line therapy in this specific subgroup of patients with known EGFR mutations.

Multiple randomized trials comparing outcomes from TKI therapy versus platinum-based regimens as first-line management of patients with NSCLC and known EGFR mutations have demonstrated promising results for recipients of the TKIs, with regard to both response rate and progression-free survival. ${ }^{36,37}$ Several studies have supported findings of an overall response rate in the range of $75 \%-80 \%$ for individuals treated with EGFR TKIs. ${ }^{29,38}$ Compared with docetaxel in a second-line setting in an open-label phase III study of individuals who were either nonsmokers or former light smokers, patients with $E G F R$ mutations achieved greater response rate and prolonged progression-free survival with gefitinib than with carboplatin in combination with paclitaxel. ${ }^{39}$

Despite the utility of this drug class, long-term results are disappointing, with most patients developing drug resistance in a matter of months, as their tumors find ways to work around the selected target. ${ }^{40}$ Exemplified by this dilemma, it is clear that the future of targeted therapy will need to rely upon strategic targeting of cancer cells in multiple ways, knocking out multiple paths and backup mechanisms for abnormal growth and proliferation.

In addition to the EGFR TKI agents, investigators have found another route of blocking EGFR activity: monoclonal antibodies have been designed to target the extracellular domain of the EGFR. Cetuximab, a chimeric human-murine immunoglobulin $\mathrm{G}$ monoclonal antibody, is one such example, and it has been examined in multiple phase II trials, in both unselected and EGFR-mutated groups of patients. ${ }^{41-43}$ Positive results from initial studies led to the undertaking of the FLEX (First-Line Treatment for Patients with EGFR-Expressing Advanced NSCLC) trial, a randomized study comparing cisplatin and vinorelbine with or without cetuximab in over 1100 patients with $E G F R$ abnormalities. ${ }^{44}$ In this study protocol, patients receiving cetuximab demonstrated a significant survival advantage compared with those who did not (11.3 versus 10.1 months, respectively; $P=0.044$ ). Panitumumab, a fully human monoclonal antibody to the EGFR, has also been evaluated, but with less favorable results. ${ }^{38}$ While early data from a phase I trial left investigators hopeful, a subsequent phase II trial of carboplatin plus paclitaxel both with and without panitumumab failed to show any difference in outcomes between the two treatment modalities for patients with previously untreated advanced NSCLC. ${ }^{45}$ Further studies are underway evaluating the efficacy of additional EGFR-targeting monoclonal antibodies such as matuzumab and nimotuzumab. ${ }^{43}$

\section{ALK}

Within the last several years, the fusion of echinoderm microtubule-associated protein-like 4 (EML4) and $A L K$ has been described and identified as a potential therapeutic target in NSCLC. ${ }^{46}$ Investigators have proposed an inversion in chromosome $2 \mathrm{p}$ leading to the formation of a rare fusion gene comprising portions of EML4 and $A L K$. This rearrangement has been noted to be more frequent among patients with adenocarcinoma of the lung and among those identified as never-smokers or light smokers. Furthermore, this derangement appears to be mutually exclusive with mutations in $E G F R$ and $K R A S .^{46-48}$ In fact, there are data to suggest that those who harbor this rearrangement are conferred no benefit at all when treated with EGFR TKIs. ${ }^{48}$

Animal studies have demonstrated tumor regression with the use of ALK inhibitors in EML4-ALK transgenic mice, rendering this target highly attractive for future drug development. ${ }^{49}$ An open-label phase II study has recently been initiated to study the efficacy of the ALK inhibitor PF-02341066, also known as crizotinib, in patients with ALK-positive advanced-stage NSCLC. ${ }^{15,28}$ Early results have shown positive objective responses, with disease control in nearly $80 \%$ at 8 weeks. ${ }^{50}$ Consequently, a phase III trial is underway, with the objective of comparing crizotinib with standard of care chemotherapy in patients with ALKpositive advanced-stage NSCLC and disease progression during treatment with platinum-based chemotherapy. ${ }^{28}$ Such strategies, if proven efficacious, may be of potential benefit to the approximately $5 \%-6 \%$ of patients with NSCLC who demonstrate this abnormality.

\section{KRAS}

$K R A S$, like other members of the RAS family of oncogenes, encodes a guanosine triphosphate-binding protein involved 
in cellular growth, differentiation, and apoptosis by interacting with multiple effectors. While KRAS mutations have been known to occur in NSCLC for over 2 decades, clinical applicability of this knowledge has been an area of more recent development. ${ }^{51}$ Among patients with NSCLC, mutations in KRAS have been identified more commonly among Caucasians, smokers (26\%, versus $6 \%$ of neversmokers), and patients with adenocarcinoma histology $(30 \%$, versus $5 \%$ with squamous carcinoma histology). ${ }^{51-54}$

$K R A S$ mutations are typically mutually exclusive of $E G F R$ mutations, occurring in different patient populations. ${ }^{48,51}$ Significant attention has been directed toward identifying the predictive and prognostic role of KRAS status in terms of response to EGFR inhibitors for the treatment of NSCLC. Initial reports have suggested that patients with NSCLC and $K R A S$ mutations might be intrinsically resistant to treatment with EGFR TKIs. ${ }^{55}$ However, it is not clear that these patients are any less susceptible than those individuals who are otherwise $E G F R$ wild-type, irrespective of $K R A S$ status. ${ }^{56,57}$ While investigators have observed an association between the presence of $K R A S$ mutations and lack of response to EGFR TKIs, the question remains as to whether there is an association between KRAS mutation status and progression-free and overall survival when treated with this class of drug. ${ }^{58}$

There are currently no existing therapies specifically targeting mutant KRAS in the treatment of NSCLC. There are outstanding preclinical models that are very well characterized and which are providing insight into $K R A S$ driven NSCLC. ${ }^{59}$ The therapeutic testing of small molecule inhibitors in these models will provide important preclinical information. Potential alternative therapeutic strategies in this direction are being pursued, with particular efforts directed toward the inhibition of downstream effector pathways such as PI3K/AKT/mTOR and RAS/RAF/MEK. ${ }^{60,61}$

\section{Additional targets}

Patients with NSCLC are now routinely screened for $E G F R$ mutations, and knowing a patient's $A L K$ and $K R A S$ status may be additionally useful in determining clinical strategy. With the capability of screening for many more genetic abnormalities, the task at hand becomes determination of those aberrations that are clinically relevant.

In a recent trial using a multiplex polymerase chain reaction (PCR)-based assay in combination with fluorescence in situ hybridization (FISH), evaluation of more than 500 NSCLC tumors was undertaken in order to elucidate genotype abnormalities. ${ }^{24}$ In addition to the more commonly screened mutations, investigators identified a small subset of patients with mutations in BRAF; phosphoinositide3-kinase, catalytic, alpha polypeptide; and HER2. While these individuals account for only $5 \%$ of the total patients screened, their tumors may be susceptible to relevant targeted therapies. ${ }^{24,62}$

\section{Diagnostic testing modalities}

Application of currently available tailored therapeutic agents requires accurate and reliable mechanisms for detection of known targetable mutations. To test for EGFR mutations, there are essentially two currently employed strategies: PCR amplification of specific mutations and comprehensive sequencing between exons 18 and $21 .^{63-65}$ In comparing these two strategies, mutation-specific PCR testing carries the risk of missing mutations, a problem that may be avoided by more complete sequencing. ${ }^{64} \mathrm{~A}$ variety of molecular diagnostic methodologies is available for $K R A S$ mutation analysis, including DNA sequencing, capillary electrophoresis, amplification refractory mutation system, and high resolution melting analysis. ${ }^{66-68}$ While direct sequencing has been considered the gold standard test, new options such as biochip-type assays are being explored. ${ }^{65,66}$ FISH is the current gold standard method used for detection of $A L K$ rearrangement among patients with NSCLC. This method involves the use of commercially available probes that flank the highly conserved translocation breakpoint within $A L K$, with differential signal observation based on the presence of translocation. ${ }^{63}$ However, recent studies have suggested that immunohistochemistry (IHC) may be a valid, practical screening tool to determine which patients may be candidates for ALK inhibitor therapy, with the more expensive FISH assay reserved for confirmation following an abnormal IHC finding. ${ }^{69}$ Reverse Transcription (RT)-PCR and multiplex assays have also been described for detection of $A L K$ rearrangement, but they appear to be less feasible than FISH or IHC. ${ }^{63}$ While these are the dominant tests in use at this time for mutation evaluation in NSCLC, new assays are constantly in development, and, as detection strategies evolve, diverse options will continue to become more widely available and cost-effective.

\section{Drug resistance in NSCLC}

Multidrug resistance (MDR) refers to the development of characteristics rendering a tumor to be unaffected by a variety of anticancer agents that are structurally and mechanistically unrelated - posing a significant dilemma in chemotherapeutic strategy. ${ }^{70}$ One proposed mechanism of MDR relates to adenosine triphosphate-binding cassette $(\mathrm{ABC})$ transporters 
as vehicles for extruding drugs from tumor cells. ${ }^{71}$ The ABC transporters comprise a superfamily of transmembrane proteins, with major members including $\mathrm{ABC}$ subfamily $\mathrm{B}$ member 1 (permeability glycoprotein), ABC subfamily $C$ members (also known as MDR-associated proteins), and ABC subfamily G member 2 (breast cancer resistance protein). ${ }^{71,72}$ Each of these transporters is known to be related to the extracellular transportation of a variety of chemotherapeutic drugs, and, recently, data have suggested that Pgb and BCRP may affect some targeted therapies for NSCLC, including EGFR TKIs. ${ }^{73,74}$ As substrates of the ABC transporters, the efficacy of the EGFR TKI drug class is subject to potentially serious clinical limitations. Additionally, a number of point mutations in the EGFR tyrosine kinase have been described as mechanisms for resistance to EGFR TKIs, allowing patients with NSCLC to either be initially unresponsive or develop secondary unresponsiveness to these agents. ${ }^{72,75}$

Several strategies have been suggested to overcome MDR, most classically including the coadministration of an $\mathrm{ABC}$ transporter inhibitor along with the anticancer drug. Another approach involves the encapsulation of anticancer drugs within liposomes or other nanoparticles to avoid extracellular efflux by $\mathrm{ABC}$ transporters. ${ }^{76}$ Additionally, monoclonal antibodies have been developed to target members of the ABC family. ${ }^{72,77}$ Other strategies employed to combat MDR, particularly with regard to NSCLC tumors bearing EGFR mutations, have included the development of second-generation and irreversible TKIs, which remain under investigation. ${ }^{76,78}$

\section{Tumor-targeted delivery}

As the broad scope of targeted therapy for NSCLC is discussed here, it is relevant to address the concept of tumortargeted drug delivery. Drug encapsulation via liposomes and other nanoparticles has been investigated, both as a means of combating MDR and as a mechanism of specifically directing the anticancer agents to the tumor cells. ${ }^{79}$ Preclinical studies have suggested that cisplatin administered within inhaled biotinylated-EGF-modified gelatin nanoparticles displays heightened delivery to EGFR-expressing tumor cells. ${ }^{80}$ Additional investigators have reported preliminary phase II data suggesting that nanoparticle binding may enhance delivery and efficacy of several drugs for NSCLC. ${ }^{79}$ Nanoparticle-based drug delivery systems have shown promise as a means of locally delivering drugs to NSCLC tumor cells via inhalation and allowing intratumoral accumulation. ${ }^{81}$ Phase III studies are underway to further assess such strategies. ${ }^{79}$

\section{The future of personalized therapy for NSCLC}

As understanding of the molecular biology of lung cancer expands, ability to apply targeted therapies to this disease process will likewise grow. One might postulate that as experience with targeted therapy and personalized oncology grows, the role of surgical resection may be called into question. The present authors believe surgical resection for early stage NSCLC will remain the gold standard. Surgeons should embrace the era of targeted therapy, as it may mean an actual increase in the number of surgical candidates as those with advanced disease become potentially resectable with a neoadjuvant personalized approach. Furthermore, having good samples of tissue is of critical importance for performing the analysis we need if we are to apply the concept of personalized oncology. With an increasing armamentarium of such treatment approaches, we hope to move closer to the ideal of personalized medicine.

One of the most difficult challenges in creating personalized therapy for patients with NSCLC lies in the limited information available regarding each patient's tumor biology. As new protocols are evaluated for patients with pretreated NSCLC, assessment of tumor biomarker status tends to reflect findings from tissue obtained at diagnosis, which may or may not correspond to the tumor's posttreatment biologic behavior. In a recent landmark trial, the BATTLE (Biomarker-Integrated Approaches of Targeted Therapy for Lung Cancer Elimination) study investigators prospectively obtained core needle biopsies of subjects' tumors and assessed them for specific tumor markers used to construct biomarker profiles. ${ }^{82}$ Based on these results, adaptive randomization was employed to assign patients to one of four treatment arms - selecting for each patient the strategy presumed to be of greatest potential benefit to that individual upon application of cumulative data. The BATTLE study investigators hypothesized that individual lung tumors are each driven by one predominant signaling pathway, among many normal pathways that may be deranged in lung cancer, and that the aberrant pathway could specifically be targeted in each individual. Using Bayesian adaptive randomization with identification of predictive markers and assimilation of findings into subsequent treatment assignments, this important study demonstrated the feasibility of a biopsy-driven approach to selecting treatment algorithms. Importantly, in addition to showing the achievability of such a study platform, the BATTLE trial also proved that patients who are prescribed treatment with existing drugs based on their tumor biomarkers will achieve 
greater clinical benefits than those whose treatments are assigned without regard to biomarker status.

Moving forward, attempts are being made to determine comprehensive genetic and biologic characterization of individuals' NSCLC tumors and to incorporate these findings into everyday practice. Using a combination of PCR and FISH assays to simultaneously identify more than 50 mutations in several key genes, investigators in a recent large-scale study used results of these tests to steer patients with advanced disease toward genotype-directed targeted therapies. ${ }^{24}$ In addition, the researchers found the results of these assays useful in distinguishing multiple primary cancers from metastatic disease. In another promising study, authors used a well-established murine model to examine the problem of metastasis in NSCLC, ultimately deriving gene signatures that characterized and successfully predicted the progression to nodal and distant metastases. ${ }^{83}$ By integrating gene expression with proteomic data, the authors further aimed to identify functionally significant molecular alterations that could be specifically targeted for therapy - facilitating a more personalized approach.

Another recent study described the use of a five-gene and corresponding protein signature to identify the patients with stage I adenocarcinoma of the lung with poor prognosis who were most likely to benefit from adjuvant therapy; such a strategy would allow clinicians to treat such individuals before postoperative recurrence. ${ }^{84}$ The potential for clinical application of such information is formidable, and the possible benefit to individual patients is immense.

Even with efforts directed toward comprehensive characterization of the genetic and molecular aberrancies in a given patient's tumor, ongoing challenges continue to be faced in the realm of targeted therapy. Approaches to personalized medicine frequently rely on single tumor biopsies to determine the optimal treatment strategy. While consideration has been given to identifying multiple potential therapeutic targets on the basis of such individual tumor samples, tumor heterogeneity remains a significant concern. In a variety of malignancies, previous investigators have noted genetic differences between primary tumors and subsequent lesions associated with local recurrence and distant metastasis. ${ }^{85-87}$ Further, additional recent studies have shown intratumor genetic heterogeneity, with varying characteristics throughout different regions of primary tumors. ${ }^{88}$ In a study evaluating tumors from patients with renal cell carcinoma, authors found mutational intratumor heterogeneity with regard to multiple tumor-suppressor genes converging on loss of function. ${ }^{88}$ These genes were found to

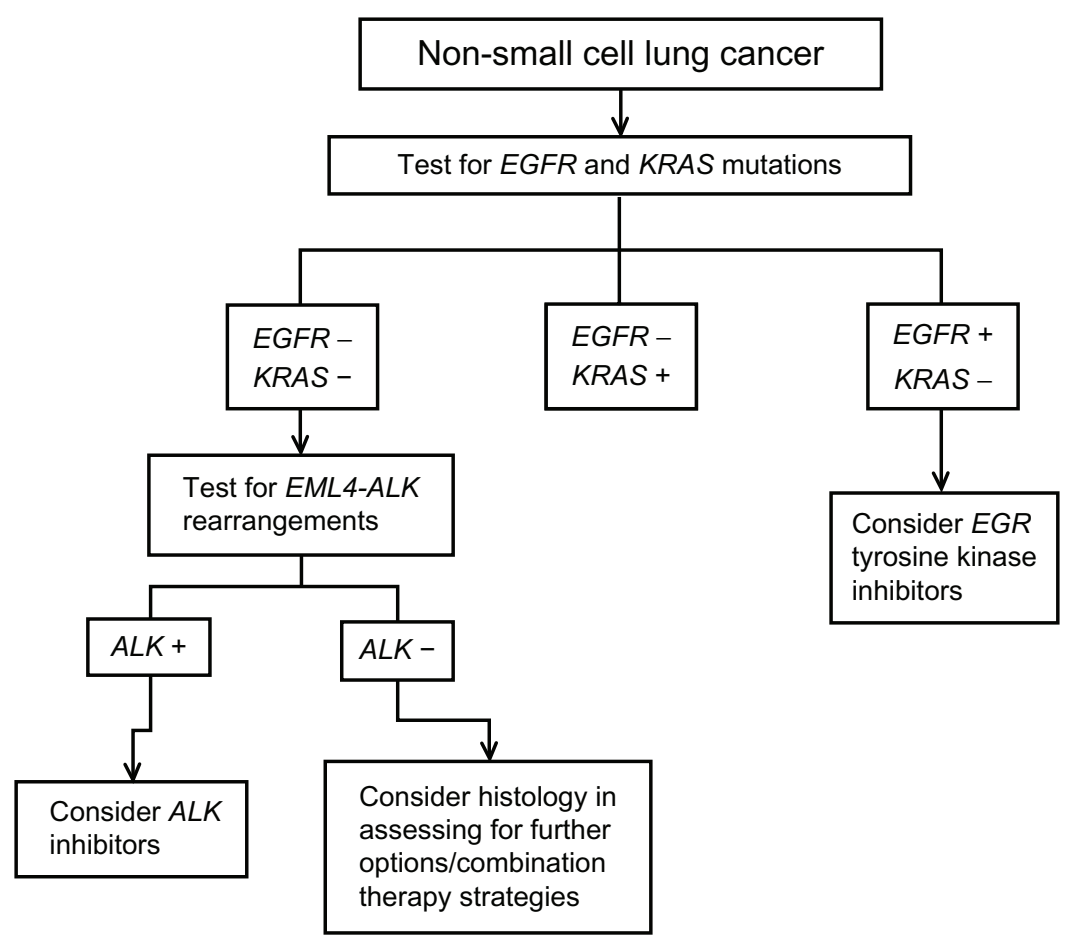

Figure 2 Proposed algorithm for non-small cell lung cancer.

Note: This schema demonstrates a proposed algorithm for genetic mutational testing and corresponding treatment strategies in non-small cell lung cancer.

Abbreviations: ALK, anaplastic lymphoma kinase; EGFR, epidermal growth factor receptor; EML4-ALK, echinoderm microtubule-associated protein-like 4 and anaplastic lymphoma kinase; KRAS, Kirsten ras. 
undergo multiple distinct and spatially separated inactivating mutations within a single tumor. Further, investigators were able to find gene-expression signatures associated with both good and poor prognosis in different regions of the same tumor. Such findings suggest the complexity of targeted therapy may have been somewhat underestimated, and they offer a potential explanation for therapeutic failures consequent to tumor adaptation. These issues do not negate the potential therapeutic benefits of previous discoveries and advances in this field. However, they contribute significantly to the framework for ongoing problem solving and future progression in this arena.

Thoracic oncologists have already begun the process of applying the findings described herein to a clinical context. As described earlier, previous treatment assignments occurred through a fairly standardized process: (1) diagnose, (2) stage, and (3) use histology and staging information to treat. Recently proposed algorithms take advantage of currently available targeted therapies, with one of these algorithms as follows: (1) test for EGFR and KRAS mutations - consider EGFR TKIs on the basis of these findings; (2) test for EML4-ALK rearrangements - if present, consider ALK inhibitors as a treatment option; and (3) if neither EGFR nor EML4-ALK abnormalities are present, consider histology in assessing for further treatment options, such as bevacizumab or pemetrexed as part of combination therapy strategies (Figure 2). ${ }^{28}$ Indicative of real-time adoption of this concept, every non-squamous tumor at the present authors' institution is sent for mutation testing.

\section{Conclusion}

As one considers the enormous worldwide burden of lung cancer morbidity and mortality, reflections on the current state of management and therapeutic options can be somewhat disappointing. The notion of characterizing every patient's tumor in terms of its genetic and biologic derangements seems to be a lofty, faraway goal, and the task of further providing each patient with a personalized plan for disease management is beyond daunting. While great strides have already been made in treating patients with EGFR mutations and ALK translocations, these account for only about $20 \%$ of patients with NSCLC. ${ }^{24,25}$ However, because of the significant prevalence of this highly lethal disease, even this small fraction amounts to a remarkable number of lives that may potentially be affected by currently available targeted therapies. Of the more than 225,000 new lung cancer diagnoses projected to occur in the United States alone over the next year, approximately 45,000 of these patients will have tumors treatable by targeting
EGFR or $A L K$ - more than the number of Americans who will die from breast, colorectal, or prostate cancer during the same period of time. ${ }^{1}$ The era of targeted therapy is upon us and these studies define the potential power of molecularly driven decision-making for those patients afflicted with this devastating malignancy.

\section{Disclosure}

The authors report no conflicts of interest in this work.

\section{References}

1. Siegel R, Naishadham D, Jemal A. Cancer statistics, 2012. CA Cancer J Clin. 2012;62(1):10-29.

2. Ettinger DS, Akerley W, Bepler G, et al. Non-small cell lung cancer J Natl Compr Canc Netw. 2010;8(7):740-801.

3. American Cancer Society: Cancer Facts and Figures 2004. Atlanta, GA: 2004

4. Carr LL, Finigan JH, Kern JA. Evaluation and treatment of patients with non-small cell lung cancer. Med Clin North Am. 2011;95(6): 1041-1054.

5. Thun MJ, Lally CA, Flannery JT, Calle EE, Flanders WD, Heath CW Jr. Cigarette smoking and changes in the histopathology of lung cancer. J Natl Cancer Inst. 1997;89(21):1580-1586.

6. Carr SR, Schuchert MJ, Pennathur A, et al. Impact of tumor size on outcomes after anatomic lung resection for stage 1A non-small cell lung cancer based on the current staging system. J Thorac Cardiovasc Surg. 2012;143(2):390-397.

7. Hrubec Z, McLaughlin JK. Former cigarette smoking and mortality among US veterans: a 26 year follow-up, 1954-1980. In: Burns DM, Garfinkel L, Samet JM, editors. Changes in Cigarette-Related Disease Risks and their Implication for Prevention and Control. Bethesda (MD): US Government Printing Office; 1997:501-530.

8. Clément-Duchêne C, Vignaud JM, Stoufflet A, et al. Characteristics of never smoker lung cancer including environmental and occupational risk factors. Lung Cancer. 2010;67(2):144-150.

9. Subramanian J, Govindan R. Lung cancer in never smokers: a review. J Clin Oncol. 2007;25(5):561-570.

10. Ginsberg RJ, Rubinstein LV; for Lung Cancer Study Group. Randomized trial of lobectomy versus limited resection for T1 N0 non-small cell lung cancer. Ann Thorac Surg. 1995;60(3):615-622; discussion 622-613.

11. Okada M, Yoshikawa K, Hatta T, Tsubota N. Is segmentectomy with lymph node assessment an alternative to lobectomy for non-small cell lung cancer of 2 cm or smaller? Ann Thorac Surg. 2001;71(3):956-960; discussion 961.

12. Burdett S, Stephens R, Stewart L, et al; for NSCLC Meta-Analyses Collaborative Group. Chemotherapy in addition to supportive care improves survival in advanced non-small-cell lung cancer: a systematic review and meta-analysis of individual patient data from 16 randomized controlled trials. J Clin Oncol. 2008;26(28):4617-4625.

13. Zhu CQ, Ding K, Strumpf D, et al. Prognostic and predictive gene signature for adjuvant chemotherapy in resected non-small-cell lung cancer. J Clin Oncol. 2010;28(29):4417-4424.

14. Pfister DG, Johnson DH, Azzoli CG, et al. American Society of Clinical Oncology treatment of unresectable non-small-cell lung cancer guideline: update 2003. J Clin Oncol. 2004;22(2):330-353.

15. Janku F, Stewart DJ, Kurzrock R. Targeted therapy in non-small-cell lung cancer: is it becoming a reality? Nat Rev Clin Oncol. 2010;7(7): 401-414.

16. Fossella FV, Lynch T, Shepherd FA. Second line chemotherapy for NSCLC: establishing a gold standard. Lung Cancer. 2002;38 Suppl $4: 5-12$. 
17. Hanna N, Shepherd FA, Fossella FV, et al. Randomized phase III trial of pemetrexed versus docetaxel in patients with non-small-cell lung cancer previously treated with chemotherapy. J Clin Oncol. 2004;22(9): $1589-1597$.

18. Silvestri GA, Rivera MP. Targeted therapy for the treatment of advanced non-small cell lung cancer: a review of the epidermal growth factor receptor antagonists. Chest. 2005;128(6):3975-3984.

19. D'Cunha J. "Personalizing" therapy for non-small cell lung cancer. Semin Thorac Cardiovasc Surg. 2010;22(2):118-120.

20. Antonoff MB, D'Cunha J. The coming of age of molecular tumor profiling. Semin Thorac Cardiovasc Surg. 2011;23(1):8-9.

21. Olaussen KA, Dunant A, Fouret P, et al. DNA repair by ERCC1 in non-small-cell lung cancer and cisplatin-based adjuvant chemotherapy. N Engl J Med. 2006;355(10):983-991.

22. Druker BJ, Sawyers CL, Kantarjian H, et al. Activity of a specific inhibitor of the BCR-ABL tyrosine kinase in the blast crisis of chronic myeloid leukemia and acute lymphoblastic leukemia with the Philadelphia chromosome. $N$ Engl J Med. 2001;344(14): 1038-1042.

23. Demetri GD, von Mehren M, Blanke CD, et al. Efficacy and safety of imatinib mesylate in advanced gastrointestinal stromal tumors. $N$ Engl J Med. 2002;347(7):472-480.

24. Sequist LV, Heist RS, Shaw AT, et al. Implementing multiplexed genotyping of non-small-cell lung cancers into routine clinical practice. Ann Oncol. 2011;22(12):2616-2624.

25. Kris MG, Johnson BE, Kwiatkowski DJ. Identification of driver mutations in tumor specimens from 1,000 patients with lung adenocarcinoma: the NCI's Lung Cancer Mutation Consortium (LCMC). J Clin Oncol. 2011;29:Abstr CRA7506.

26. Boulougouris P, Elder J. Epidermal growth factor receptor structure, regulation, mitogenic signalling and effects of activation. Anticancer Res. 2001;21(4A):2769-2775.

27. Shigematsu H, Lin L, Takahashi T, et al. Clinical and biological features associated with epidermal growth factor receptor gene mutations in lung cancers. J Natl Cancer Inst. 2005;97(5):339-346.

28. Wigle DA. Personalized therapy for non-small cell lung cancer: hype or clinical reality? Semin Thorac Cardiovasc Surg. 2011;23(1):30-35.

29. Mitsudomi T, Yatabe Y. Mutations of the epidermal growth factor receptor gene and related genes as determinants of epidermal growth factor receptor tyrosine kinase inhibitors sensitivity in lung cancer. Cancer Sci. 2007;98(12):1817-1824.

30. Kris MG, Natale RB, Herbst RS, et al. Efficacy of gefitinib, an inhibitor of the epidermal growth factor receptor tyrosine kinase, in symptomatic patients with non-small cell lung cancer: a randomized trial. JAMA. 2003;290(16):2149-2158.

31. Fukuoka M, Yano S, Giaccone G, et al. Multi-institutional randomized phase II trial of gefitinib for previously treated patients with advanced non-small-cell lung cancer (the IDEAL 1 Trial) [corrected]. $J$ Clin Oncol. 2003;21(12):2237-2246.

32. Shepherd FA, Rodrigues Pereira J, Ciuleanu T, et al. Erlotinib in previously treated non-small-cell lung cancer. NEnglJMed.2005;353(2): 123-132.

33. Lynch TJ, Bell DW, Sordella R, et al. Activating mutations in the epidermal growth factor receptor underlying responsiveness of nonsmall-cell lung cancer to gefitinib. $N$ Engl J Med. 2004;350(21): 2129-2139.

34. Maemondo M, Inoue A, Kobayashi K, et al. Gefitinib or chemotherapy for non-small-cell lung cancer with mutated EGFR. $N$ Engl $J$ Med. 2010;362(25):2380-2388.

35. Lynch TJ, Fenton D, Hirsh V, et al. A randomized phase 2 study of erlotinib alone and in combination with bortezomib in previously treated advanced non-small cell lung cancer. JThorac Oncol. 2009;4(8): 1002-1009.

36. Mitsudomi T, Morita S, Yatabe Y, et al. Gefitinib versus cisplatin plus docetaxel in patients with non-small-cell lung cancer harbouring mutations of the epidermal growth factor receptor (WJTOG3405): an open label, randomised phase 3 trial. Lancet Oncol. 2010;11(2): 121-128.
37. Vilmar AC, Sorensen JB. Customising chemotherapy in advanced nonsmall cell lung cancer: daily practice and perspectives. Eur Respir Rev. 2011;20(119):45-52.

38. Gettinger S. Targeted therapy in advanced non-small-cell lung cancer. Semin Respir Crit Care Med. 2008;29(3):291-301.

39. Mok TS, Wu YL, Thongprasert S, et al. Gefitinib or carboplatinpaclitaxel in pulmonary adenocarcinoma. $N$ Engl J Med. 2009;361(10): 947-957.

40. Ma C, Wei S, Song Y. T790M and acquired resistance of EGFR TKI: a literature review of clinical reports. J Thorac Dis. 2011;3(1):10-18.

41. Thienelt CD, Bunn PA Jr, Hanna N, et al. Multicenter phase I/II study of cetuximab with paclitaxel and carboplatin in untreated patients with stage IV non-small-cell lung cancer. J Clin Oncol. 2005;23(34): 8786-8793.

42. Belani CP, Schreeder MT, Steis RG, et al. Cetuximab in combination with carboplatin and docetaxel for patients with metastatic or advancedstage nonsmall cell lung cancer: a multicenter phase 2 study. Cancer. 2008;113(9):2512-2517.

43. Gridelli C, Maione P, Ferrara ML, Rossi A. Cetuximab and other antiepidermal growth factor receptor monoclonal antibodies in the treatment of non-small cell lung cancer. Oncologist. 2009;14(6):601-611.

44. Pirker R, Pereira JR, Szczesna A, et al. Cetuximab plus chemotherapy in patients with advanced non-small-cell lung cancer (FLEX): an openlabel randomised phase III trial. Lancet. 2009;373(9674):1525-1531.

45. Socinski MA. Antibodies to the epidermal growth factor receptor in non small cell lung cancer: current status of matuzumab and panitumumab. Clin Cancer Res. 2007;13(15 Pt 2):S4597-S4601.

46. Soda M, Choi YL, Enomoto M, et al. Identification of the transforming EML4-ALK fusion gene in non-small-cell lung cancer. Nature. 2007;448(7153):561-566.

47. Choi YL, Takeuchi K, Soda M, et al. Identification of novel isoforms of the EML4-ALK transforming gene in non-small cell lung cancer. Cancer Res. 2008;68(13):4971-4976.

48. Shaw AT, Yeap BY, Mino-Kenudson M, et al. Clinical features and outcome of patients with non-small-cell lung cancer who harbor EML4-ALK. J Clin Oncol. 2009;27(26):4247-4253.

49. Soda M, Takada S, Takeuchi K, et al. A mouse model for EML4ALK-positive lung cancer. Proc Natl Acad Sci U S A. 2008;105(50): 19893-19897.

50. Kwak EL, Camidge DR, Clark J, et al. Clinical activity observed in a phase I dose escalation trial of an oral c-Met and ALK inhibitor, PF-02341066. J Cin Oncol. 2009;27:Abstr 3509.

51. Riely GJ, Marks J, Pao W. KRAS mutations in non-small cell lung cancer. Proc Am Thorac Soc. 2009;6(2):201-205.

52. Riely GJ, Kris MG, Rosenbaum D, et al. Frequency and distinctive spectrum of KRAS mutations in never smokers with lung adenocarcinoma. Clin Cancer Res. 2008;14(18):5731-5734.

53. Mao C, Qiu LX, Liao RY, et al. KRAS mutations and resistance to EGFR-TKIs treatment in patients with non-small cell lung cancer: a meta-analysis of 22 studies. Lung Cancer. 2010;69(3):272-278.

54. Graziano SL, Gamble GP, Newman NB, et al. Prognostic significance of K-Ras codon 12 mutations in patients with resected stage I and II non-small-cell lung cancer. J Clin Oncol. 1999;17(2):668-675.

55. Pao W, Wang TY, Riely GJ, et al. KRAS mutations and primary resistance of lung adenocarcinomas to gefitinib or erlotinib. PLoS Med. 2005;2(1):e17.

56. Jackman DM, Miller VA, Cioffredi LA, et al. Impact of epidermal growth factor receptor and KRAS mutations on clinical outcomes in previously untreated non-small cell lung cancer patients: results of an online tumor registry of clinical trials. Clin Cancer Res. 2009;15(16): 5267-5273.

57. Gaughan EM, Costa DB. Genotype-driven therapies for non-small cell lung cancer: focus on EGFR, KRAS and ALK gene abnormalities. Ther Adv Med Oncol. 2011;3(3):113-125.

58. Roberts PJ, Stinchcombe TE, Der CJ, Socinski MA. Personalized medicine in non-small-cell lung cancer: is KRAS a useful marker in selecting patients for epidermal growth factor receptor-targeted therapy? J Clin Oncol. 2010;28(31):4769-4777. 
59. DuPage M, Dooley AL, Jacks T. Conditional mouse lung cancer models using adenoviral or lentiviral delivery of Cre recombinase. Nat Protoc. 2009;4(7):1064-1072.

60. Engelman JA, Chen L, Tan X, et al. Effective use of PI3K and MEK inhibitors to treat mutant Kras G12D and PIK3CA H1047R murine lung cancers. Nat Med. 2008;14(12):1351-1356.

61. Sos ML, Fischer S, Ullrich R, et al. Identifying genotype-dependent efficacy of single and combined PI3K- and MAPK-pathway inhibition in cancer. Proc Natl Acad Sci U S A. 2009;106(43):18351-18356.

62. Pao W, Girard N. New driver mutations in non-small-cell lung cancer. Lancet Oncol. 2011;12(2):175-180.

63. Shaw AT, Hayes DN, Martins R. The importance of histology and molecular testing (EGFR and EML4-ALK) in the initial evaluation of advanced non-small cell lung cancer. ASCO Education Book. 2011: 292-298.

64. Penzel R, Sers C, Chen Y, et al. EGFR mutation detection in NSCLC: assessment of diagnostic application and recommendations of the German Panel for Mutation Testing in NSCLC. Virchows Arch. 2011; 458(1):95-98.

65. Tanner NT, Pastis NJ, Sherman C, Simon GR, Lewin D, Silvestri GA. The role of molecular analyses in the era of personalized therapy for advanced NSCLC. Lung Cancer. 2012;76(2):131-137.

66. Kriegshäuser G, Fabjani G, Ziegler B, Zöchbauer-Müller S, End A, Zeillinger R. Biochip-based detection of KRAS mutation in non-small cell lung cancer. Int J Mol Sci. 2011;12(12):8530-8538.

67. Kotoula V, Charalambous E, Biesmans B, et al. Targeted KRAS mutation assessment on patient tumor histologic material in real time diagnostics. PLoS One. 2009;4(11):e7746.

68. Krypuy M, Newnham GM, Thomas DM, Conron M, Dobrovic A. High resolution melting analysis for the rapid and sensitive detection of mutations in clinical samples: KRAS codon 12 and 13 mutations in non-small cell lung cancer. BMC Cancer. 2006;6:295.

69. Park HS, Lee JK, Kim DW, et al. Immunohistochemical screening for anaplastic lymphoma kinase (ALK) rearrangement in advanced nonsmall cell lung cancer patients. Lung Cancer. Epub March 30, 2012.

70. Laurent-Puig P, Lievre A, Blons H. Mutations and response to epidermal growth factor receptor inhibitors. Clin Cancer Res. 2009;15(4): 1133-1139.

71. Scagliotti GV, Novello S, Selvaggi G. Multidrug resistance in nonsmall-cell lung cancer. Ann Oncol. 1999;10 Suppl 5:S83-S86.

72. Colabufo NA, Contino M, Niso M, Berardi F, Leopoldo M, Perrone R. EGFR tyrosine kinase inhibitors and multidrug resistance: perspectives. Front Biosci. 2011;16:1811-1823.

73. Shi Z, Tiwari AK, Shukla S, et al. Inhibiting the function of ABCB1 and ABCG2 by the EGFR tyrosine kinase inhibitor AG1478. Biochem Pharmacol. 2009;77(5):781-793.

74. Dai CL, Tiwari AK, Wu CP, et al. Lapatinib (Tykerb, GW572016) reverses multidrug resistance in cancer cells by inhibiting the activity of ATP-binding cassette subfamily B member 1 and G member 2. Cancer Res. 2008;68(19):7905-7914.
75. Regales L, Balak MN, Gong Y, et al. Development of new mouse lung tumor models expressing EGFR T790M mutants associated with clinical resistance to kinase inhibitors. PLoS One. 2007;2(8):e810.

76. Li D, Ambrogio L, Shimamura T, et al. BIBW2992, an irreversible EGFR/HER2 inhibitor highly effective in preclinical lung cancer models. Oncogene. 2008;27(34):4702-4711.

77. Colabufo NA, Berardi F, Cantore M, et al. Perspectives of P-glycoprotein modulating agents in oncology and neurodegenerative diseases: pharmaceutical, biological, and diagnostic potentials. J Med Chem. 2010;53(5):1883-1897.

78. Milojkovic D, Apperley J. Mechanisms of resistance to imatinib and second-generation tyrosine inhibitors in chronic myeloid leukemia. Clin Cancer Res. 2009;15(24):7519-7527.

79. Stewart DJ. Tumor and host factors that may limit efficacy of chemotherapy in non-small cell and small cell lung cancer. Crit Rev Oncol Hematol. 2010;75(3):173-234.

80. Tseng CL, Su WY, Yen KC, Yang KC, Lin FH. The use of biotinylatedEGF-modified gelatin nanoparticle carrier to enhance cisplatin accumulation in cancerous lungs via inhalation. Biomaterials. 2009; 30(20):3476-3485.

81. Taratula O, Garbuzenko OB, Chen AM, Minko T. Innovative strategy for treatment of lung cancer: targeted nanotechnology-based inhalation co-delivery of anticancer drugs and siRNA. J Drug Target. 2011;19(10): 900-914.

82. Kim ES, Herbst RS, Wistuba II, et al. The BATTLE Trial: personalizing therapy for lung cancer. Cancer Discovery. 2011;1(1):44-53.

83. Carretero J, Shimamura T, Rikova K, et al. Integrative genomic and proteomic analyses identify targets for Lkb1-deficient metastatic lung tumors. Cancer Cell. 2010;17(6):547-559.

84. Kadara H, Behrens C, Yuan P, et al. A five-gene and corresponding protein signature for stage-I lung adenocarcinoma prognosis. Clin Cancer Res. 2011;17(6):1490-1501.

85. Ding L, Ellis MJ, Li S, et al. Genome remodelling in a basal-like breast cancer metastasis and xenograft. Nature. 2010;464(7291):999-1005.

86. Yachida S, Jones S, Bozic I, et al. Distant metastasis occurs late during the genetic evolution of pancreatic cancer. Nature. 2010;467(7319): 1114-1117.

87. Tao Y, Ruan J, Yeh SH, et al. Rapid growth of a hepatocellular carcinoma and the driving mutations revealed by cell-population genetic analysis of whole-genome data. Proc Natl Acad Sci U S A. 2011;108(29):12042-12047.

88. Gerlinger M, Rowan AJ, Horswell S, et al. Intratumor heterogeneity and branched evolution revealed by multiregion sequencing. $N$ Engl J Med. 2012;366(10):883-892.
Lung Cancer: Targets and Therapy

\section{Publish your work in this journal}

Lung Cancer: Targets and Therapy is an international, peer-reviewed, open access journal focusing on lung cancer research, identification of therapeutic targets and the optimal use of preventative and integrated treatment interventions to achieve improved outcomes, enhanced survival and quality of life for the cancer patient. Specific topics covered in the journal include:

\section{Dovepress}

Epidemiology, detection and screening; Cellular research and biomarkers; Identification of biotargets and agents with novel mechanisms of action; Optimal clinical use of existing anticancer agents, including combination therapies; Radiation and surgery; Palliative care; Patient adherence, quality of life, satisfaction; Health economic evaluations. 